\title{
RIGID PAIRS OF LONG ARCS ${ }^{1}$
}

\author{
GARY G. MILLER ${ }^{2}$
}

\begin{abstract}
Arcs (linearly ordered continua) $A$ and $B$ are constructed such that every map from $A$ to $B$ and every map from $B$ to $A$ is constant. The Generalized Continuum Hypothesis is sufficient for the existence of two such arcs each of cardinality $2^{\boldsymbol{N}}$ for each uncountable cardinal $\boldsymbol{~}$.
\end{abstract}

1. Introduction. By an arc we mean a compact connected Hausdorff space with exactly two noncut points. A separable arc is then a homeomorph of the real interval $I=[0,1]$. An arc is long if it is not separable or equivalently not metrizable. A survey of the literature on long arcs is given in [2].

Here we construct arcs $A$ and $B$ such that if $f: A \rightarrow B$ and $g: B \rightarrow A$ are continuous, then the image of each function is a point. We say such a pair of arcs is rigid. Due to Urysohn's lemma, neither $A$ nor $B$ contains a separable arc. Moreover if $C$ and $D$ are subarcs of a rigid pair of arcs $A$ and $B$ respectively, then $C$ and $D$ form a rigid pair. This follows immediately since subarcs are retracts. The desired arcs are obtained by constructing two kinds of simply ordered sets.

Let $X$ be a simply ordered set (chain) with at least two elements. The intrinsic topology of $X$ is obtained by taking open intervals and open rays as a basis. $X$ is then an arc if $X$ has a supremum, every nonempty subset of $X$ has an infimum, and for any two points $x<y$ there is a point $z, x<z<y$ $[1, X, \S \S 7-8]$.

2. Arcs from sequences of ordinals. The usual construction of the unit interval from sequences of digits involves the identification of discontinuities, e.g., $0.4999 \cdots$ and $0.5000 \cdots$. In this section, long arcs are constructed in somewhat the same fashion from sequences of ordinals. Here identification is unnecessary.

Received by the editors January 19, 1971.

AMS 1970 subject classifications. Primary 54C20, 54F05, 54G15; Secondary 06A05, 54A25, 54B99, 54F50.

Key words and phrases. Arcs, linearly ordered continua, continuous functions, Urysohn's lemma, lexicographic product, ordinal power, rigid pairs of spaces.

${ }^{1}$ This research was supported in part by the National Research Council of Canada under Grant No. A7571.

${ }^{2}$ The author wishes to thank B. J. Pearson and C. A. Eberhart for their comments. 
Let $\omega$ be the first infinite ordinal and let $\lambda$ be any limit ordinal. Then $\lambda^{\omega}$ denotes the set of all sequences of ordinals less than $\lambda$. Order $\lambda^{\omega}$ lexicographically, i.e., $x(0), x(1), \cdots<y(0), y(1), \cdots$ if and only if there is an $n$ such that $x(n)<y(n)$ and $x(i)=y(i)$ for all $i<n$. A discussion of such ordinal powers may be found in [1]. The simply ordered set obtained by adjoining a supremum 1 to $\lambda^{\omega}$ is denoted $\lambda^{\omega}+1$.

THEOREM 1. $\lambda^{\omega}+1$ is an arc.

Proof. Suppose $M$ is a nonempty subset of $\lambda^{\omega}$. Let $m(0)$ be the infimum of the set of first terms of sequences in $M$. Assume further $m(i)$ is defined for all $i<n$. Then let $m(n)$ be the infinimum of the set of $n$th terms of sequences in $M$ whose first $n-1$ terms are $m(0), m(1), \cdots, m(n-1)$. The sequence $m(0), m(1), \cdots, m(n), \cdots$ is the infimum of $M$ in $\lambda^{\omega}$. Therefore every nonempty subset of $\lambda^{\omega}+1$ has an infimum. Now suppose $x, y \in \lambda^{\omega}+1$ and $x<y$. Assume $y=1$. Since $\lambda$ is a limit ordinal, $x(0)$ has successor $x(0)+1<\lambda$. Let $z$ be the sequence $x(0)+1,0,0,0, \cdots$. Then $x<z<y$. Finally assume $y \in \lambda^{\omega}$. Then there is an $n$ such that $x(n)<y(n)$ and $x(i)=y(i)$ for all $i<n$. Let $z=x(0), x(1), \cdots, x(n), x(n+1)+1,0,0,0, \cdots$. Then $x<z<y$. This completes the proof.

COROLlARY. The nonnegative reals are isomorphic to the set of all sequences of nonnegative integers ordered lexicographically.

THEOREM 2. $\lambda^{\omega}$ contains a subset $D$ such that each point of the space is the limit of a descending sequence of points of $D$.

Proof. Let $D$ be the set of all $x$ such that $x$ is eventually the ordinal 0 , i.e., such that there is an $n<\omega$ and $x(m)=0$ for all $m, n<m<\omega$. Suppose $x \in \lambda^{\omega}$. For each $n<\omega$, let $d_{n}$ be the following point of $D . d_{n}(m)=x(m)$ for all $m<n, d_{n}(n)$ is the successor of $x(n)$, and $d_{n}(m)=0$ for all $m>n$. Then $d_{n}>d_{n+1}>x$ and $\operatorname{limit}\left\{d_{n}\right\}=x$.

If $X$ is a set, we denote the cardinal of $X$ by $\aleph(X)$.

TheOREM 3. Every subarc of $\lambda^{\omega}$ contains $\aleph(\lambda)$ pairwise disjoint open sets.

Proof. Let $x, y \in \lambda^{\omega}+1$ such that $x<y$. Then there is an $n<\omega$ such that $x(n)<y(n)$ and $x(i)=y(i)$ for all $i<n$. For each $\alpha \in \lambda$, let $U_{\alpha}$ be the open interval $\left(\alpha_{1}, \alpha_{2}\right)$ where $\alpha_{1}(i)=x(i)$ for all $i<n, \alpha_{1}(n+1)=x(n+1)+1$, $\alpha_{1}(n+2)=\alpha, \alpha_{1}(i)=0$ for all $i>n+3, \alpha_{1}(i)=\alpha_{2}(i)$ for all $i \neq n+3, \alpha_{1}(n+3)=$ 0 and $\alpha_{2}(n+3)=1$. Then $\left\{U_{\alpha}\right\}$ is a collection of pairwise disjoint open intervals each in $(x, y)$, the cardinal of this collection is $N(\lambda)$.

3. Ordinal powers of the unit interval. V. Novák [4] has shown that $I^{\mu}$ is an arc for any ordinal $\mu$. Here $I^{\mu}$ is the set of all functions from $\mu$ to $I$ ordered lexicographically. 
Let $\mu$ be any limit ordinal such that for all $\alpha<\mu, \aleph(\alpha)<\aleph(\mu)$.

THEOREM 4. $I^{\mu}$ contains a dense subset $M$ such that every subset with a cluster point in $M$ has cardinality at least $\aleph(\mu)$.

Proof. Let $M=\{x$ : there is an $\alpha<\mu$ such that $0<x(\beta)<1$ for all $\beta, \alpha<\beta<\mu\}$. Suppose $a, b \in I^{\mu}$ and $a<b$. Let $\alpha$ be the first ordinal such that $a(\alpha)<b(\alpha)$. Let $C \in I$ such that $a(\alpha)<C<b(\alpha)$. Define $x$ by $(1) x(\beta)=a(\beta)$ for all $\beta<\alpha$, and (2) $x(\beta)=C$ for all $\beta, \alpha<\beta<\mu$. Then $x \in M$ and $a<x<b$. Therefore $M$ is a dense subset of $I^{\mu}$.

Now suppose $X$ is a subset of $I^{\mu}, x \in M \cap X$ and each neighborhood of $x$ contains a point of $X$ distinct from $x$. Index $X-x$ so that points are not indexed twice, $X-x=\left\{x_{\gamma}: \gamma<\zeta\right\}$. Define $\psi: \zeta \rightarrow \mu$ as follows. For each $\gamma<\zeta$, let $\psi(\gamma)$ be the first $\eta<\mu$ such that $x(\eta) \neq x_{\gamma}(\eta)$. Suppose there is a $\delta<\mu$ such that $\psi(\gamma)<\delta$ for all $\gamma<\zeta$. Let $\alpha<\mu$ be an ordinal such that $0<x(\beta)<1$ for all $\beta, \alpha<\beta<\mu$. Let $a, b \in I^{\mu}$ such that $a(\gamma)=b(\gamma)=x(\gamma)$ for all $\gamma<\max [\alpha, \delta]$ and $a(\max [\alpha, \delta])<x(\max [\alpha, \delta])<b(\max [\alpha, \delta])$. Then the interval $(a, b)$ is a neighborhood of $x$ which contains no point of $X$ distinct from $x$. This involves a contradiction. Thus image $\psi$ is cofinal with $\mu$. Therefore $\aleph(\zeta) \geqq \aleph(\mu)$. This completes the proof.

THEOREM 5. $I^{\mu}$ contains a dense subset with cardinality $\sum_{\alpha<\mu} 2^{\boldsymbol{\aleph}_{0} \cdot \boldsymbol{\aleph}(\mu)}$.

Proof. Let $D$ be the set of all points which are eventually 0 . Then $D$ is a dense subspace of $I^{\mu}$.

$$
\aleph(D)=\aleph\left(\bigcup_{\alpha<\mu} I^{\alpha}\right)=\sum_{\alpha<\mu} \aleph\left(I^{\alpha}\right)=\sum_{\alpha<\mu} 2^{\aleph_{0} \cdot \aleph(\alpha)} .
$$

4. The main result. Let $\mu$ and $\lambda$ be any limit ordinals such that $\mu$ is uncountable and $\aleph(\lambda)>\sum_{\alpha<\mu} 2^{\boldsymbol{N}(\alpha)}$. If we assume the Continuum Hypothesis, we could take $\mu=\Omega$, the first uncountable ordinal, and $\lambda$ the first ordinal such that $\aleph(\lambda)>2^{\aleph_{0}}$.

THEOREM 6. The arcs $I^{\mu}$ and $\lambda^{\omega}+1$ form a rigid pair.

Proof. Suppose $f$ is a nonconstant map from $I^{\mu}$ to $\lambda^{\omega}+1$. By Theorem $5, I^{\mu}$ contains a dense subset $D$ with $\aleph(D)=\sum_{\alpha<\mu} 2^{\aleph_{0} \cdot \boldsymbol{\kappa}(\alpha)}=\sum_{\alpha<\mu} 2^{\kappa(\alpha)}<$ $\boldsymbol{\aleph}(\lambda)$. Therefore $f[D]$ is a dense subset of some subarc of $\lambda^{\omega}+1$. By Theorem $3, \boldsymbol{\aleph}(\lambda) \leqq \aleph(f[D]) \leqq \aleph(D)$. This involves a contradiction.

Finally suppose $f$ is a nonconstant map from $\lambda^{\omega}+1$ to $I^{\mu}$. Let $M$ be as in Theorem 4. Select a point $x$ in $M \cap$ image $f$ which is not the image of 1 . Then $f^{-1}(x)$ is a compact subset of $\lambda^{\omega}$. Thus $y=\operatorname{supremum} f^{-1}(x)$ is in $f^{-1}(x)$. By Theorem 2, there is a descending sequence $\left\{d_{n}: n<\omega\right\}$ in $\lambda^{\omega}$ with limit $y$. Let $X=\left\{f\left(d_{n}\right): n<\omega\right\}$. Then $x$ is a cluster point of $X$. By Theorem $4, \aleph(X) \geqq \aleph(\mu)$. This involves a contradiction. 
5. Other rigid pairs. If we assume the Generalized Continuum Hypothesis, there are two arcs of arbitrarily large cardinality that form a rigid pair.

THEOREM 7. For each uncountable cardinal $\boldsymbol{\aleph}$, there are two arcs each of cardinality $2^{\mathrm{N}}$ that form a rigid pair.

Proof. Let $\mu$ and $\lambda$ be the first ordinals such that $\boldsymbol{\aleph}(\mu)=\boldsymbol{\aleph}$ and $\boldsymbol{\aleph}(\lambda)=2^{\aleph}$. $\boldsymbol{\aleph}(\mu)=\sum_{\alpha<\mu} 2^{\boldsymbol{N}^{(\alpha)}}$. Therefore $I^{\mu}$ and $\lambda^{\omega}+1$ are two rigid arcs. $\boldsymbol{\aleph}\left(I^{\mu}\right)=2^{\boldsymbol{\kappa}_{0} \cdot \kappa}=2^{\boldsymbol{\kappa}}$ and $\boldsymbol{\aleph}\left(\lambda^{\omega}+1\right)=2^{\boldsymbol{\kappa} \cdot \boldsymbol{\kappa}_{0}}=2^{\boldsymbol{\kappa}}$.

An arc is strongly homogeneous provided it is homeomorphic to each of its subarcs. For references and a discussion of strongly homogeneous arcs, see [2]. Every such arc has cardinality $2^{\aleph_{0}}$. Therefore no arc in one of the previous rigid pairs is strongly homogeneous. However if $\mu$ is any limit ordinal such that every final segment has order type $\mu$, then every subarc of $I^{\mu}$ contains a subarc homeomorphic to $I^{\mu}$.

Problem 1. Is there a rigid pair of strongly homogeneous arcs?

Problem 2. Are there three arcs such that any two form a rigid pair?

Problem 3. Given an arbitrary cardinal $\boldsymbol{\aleph}$, are there $\boldsymbol{N}$ arcs such that any two form a rigid pair?

\section{REFERENCES}

1. G. Birkhoff, Lattice theory, 3rd ed., Amer. Math. Soc. Colloq. Publ., vol. 25, Amer. Math. Soc., Providence, R.I., 1967. MR 37 \#2638.

2. M. A. Maurice, Compact ordered spaces, Mathematical Centre Tracts, 6, Mathematisch Centrum, Amsterdam, 1964. MR 36 \#3318.

3. G. Miller Jumps and gaps in lexicographic products (to appear).

4. V. Novák, On the lexicographic dimension of linearly ordered sets, Fund. Math. 56 (1964), 9-20. MR 34 \#7412.

Department of Mathematics, University of Victoria, Victoria, British Columbia, Canada 